\title{
What can the outskirts of galaxies tell us about dark matter?
}

\author{
Chris Power \\ International Centre for Radio Astronomy Research, The University of Western Australia, 35 \\ Stirling Highway, Crawley, Western Australia 6009, Australia \\ E-mail: chris.power@icrar.org
}

\begin{abstract}
Deep observations of galaxy outskirts reveal faint extended stellar components (ESCs) of streams, shells, and halos, which are ghostly remnants of the tidal disruption of satellite galaxies. We use cosmological galaxy formation simulations in Cold Dark Matter (CDM) and Warm Dark Matter (WDM) models to explore how the dark matter model influences the spatial, kinematic, and orbital properties of ESCs. These reveal that the spherically averaged stellar mass density at large galacto-centric radius can be depressed by up to a factor of $\sim 10$ in WDM models relative to the CDM model, reflecting the anticipated suppressed abundance of satellite galaxies in WDM models. However, these differences are much smaller in WDM models that are compatible with observational limits, and are comparable in size to the systemto-system variation we find within the CDM model. This suggests that it will be challenging to place limits on dark matter using only the unresolved ESC.
\end{abstract}

Keywords. galaxies: formation — galaxies: evolution — dark matter — methods: numerical

\section{Motivation}

The defining prediction of the Cold Dark Matter (CDM) model of cosmological structure formation is that dark matter haloes contain an abundance of lower mass substructure haloes (hereafter subhalos; e.g. Klypin et al. 1999, Moore et al. 1999). Alternatives to the CDM model, such as Warm Dark Matter (WDM), suppress the abundance of lowmass dark matter halos, and consequently the abundance of subhalos, but distinguishing between these alternatives and CDM in this way in a robust fashion is challenging (e.g. Power 2013). This is because the mass scale at which differences between plausible dark matter models is most likely to be evident - at or below the scale of the satellites of the Milky Way (e.g. Schneider et al. 2014) - is also the mass scale at or below which galaxy formation is inefficient and apparently stochastic (e.g. Boylan-Kolchin et al. 2011).

However, deep observations of the Milky Way and external galaxies reveal that they are embedded in a diffuse, extended stellar component (ESC) of shells, streams, and halos (e.g. Freeman \& Bland-Hawthorn 2002, Helmi 2008), which are the relics of the tidal disruption of satellite galaxies (e.g. Bullock \& Johnston 2005, Cooper et al. 2010). Because these satellites are embedded within low-mass dark matter subhalos, it is plausible that the underlying dark matter model could leave an imprint on the structure of the ESC - models in which the abundance of low-mass subhalos, and consequently satellite galaxies, is suppressed could result in less massive, less luminous, and more centrally concentrated ESCs compared to CDM. This is because subhalos follow similar orbits in CDM and WDM models (e.g. Knebe et al. 2008), and because low-mass subhalos and satellites follow preferentially radial orbits and require many pericentric passages before their orbits decay, their tidally stripped stars can be spread over large galacto-centric distances. The physics that governs galaxy formation should not depend on the underlying dark matter model, and so differences in ESC properties should reflect differences in 
satellite abundance, which depends on the subhalo abundance. These differences could be accessible to current (e.g. van Dokkum et al. 2014) and future surveys (e.g. LSST Science Collaboration 2009) that target the diffuse, low surface brightness environs of galaxies, and so represents a potentially robust observational test of dark matter.

\section{Extended Stellar Components as an Observational Testbed for Dark Matter}

We have tested the feasibility of this idea in Power \& Robotham (2016) using a set of cosmological zoom galaxy formation simulations of 6 Milky Way mass galaxies (MW01 to MW06) forming in a CDM model, with $M_{200} \simeq 2 \times 10^{12} h^{-1} \mathrm{M}_{\odot}$ and $R_{200} \simeq 200 h^{-1} \mathrm{kpc}$ at $z=0$. These were run with a version of the GADGET $N$-body/SPH code of Springel (2005) that includes prescriptions for cooling, star formation, and supernova feedback.

In the case of one of the galaxies, MW02, we ran 3 additional versions, assuming WDM models with particle masses of $m_{\mathrm{WDM}}=0.5,1$, and $2 \mathrm{keV} / c^{2}$; this was done by modifying the initial CDM power spectrum following the approach of Bode et al. (2001). Although models with $m_{\mathrm{WDM}}<2 \mathrm{keV} / c^{2}$ are not favoured by current observational limits, we wanted to establish whether or not dark matter models that alter the abundance of substructure can leave an imprint on observable properties of galaxies.

These simulations reveal that it is in the spatial structure of the ESC that the imprint of the underlying dark matter model is most readily apparent. In the left hand panel of Figure 1, we show spherically averaged mass density profiles at $z=0$ for stars, gas, and dark matter for the the CDM and WDM $m_{\mathrm{WDM}}=2,1$, and $0.5 \mathrm{keV} / c^{2} \mathrm{keV}$ versions of MW02 (dotted-dashed, dotted, dashed, solid curves), while in the right hand panel we show only stellar profiles for the CDM versions of the 6 galaxies, MW01 to MW06. This Figure confirms our expectation that properties of the ESC varies with the underlying dark matter model - outside of the region within which the galaxy disc reside, between $R / R_{200} \sim 0.1$ and $R / R_{200} \sim 0.5$, we find that the spherically averaged stellar density is approximately an order of magnitude smaller in the WDM $m_{\mathrm{WDM}}=1$ and $0.5 \mathrm{keV} / c^{2}$ runs than in the CDM and WDM $2 \mathrm{keV} / c^{2}$ runs. This difference is imprinted early in the history of the galaxy at least back to $z \simeq 4-$ and persists to $z=0$.

However, these significant differences are most readily apparent only in the more exteme WDM models that we consider, with $m_{\mathrm{WDM}}<2 \mathrm{keV} / c^{2}$; for values of $m_{\mathrm{WDM}}$ consistent with observational limits, differences with respect to the favoured CDM model are comparable in size to the galaxy-to-galaxy variation in the CDM model, as we can infer from the right panel of Figure 1. This variation, which reflect differences in assembly history, highlights the difficulty of using the ESC as a test of dark matter - but suggests that the ESC can be used to extend the concept of galactic archaeology to systems beyond the Milky Way and Andromeda, as is being done in nearby galaxies (e.g. Martinez-Delgado et al. 2010, van Dokkum et al. 2014), and will become possible for statistical samples of galaxies within the Local Volume with, for example, LSST (LSST Science Collaboration 2009). Combining metallicity, kinematics, and spatial structure, it should be possible to trace the assembly history of galaxies.

\section{Prospects}

The focus of this work has been on the utility of the unresolved ESC - the spatial distribution and kinematics of stellar material in galaxy outskirts that will be accessible to deep imaging surveys, possibly by stacking large numbers of galaxies by central galaxy 

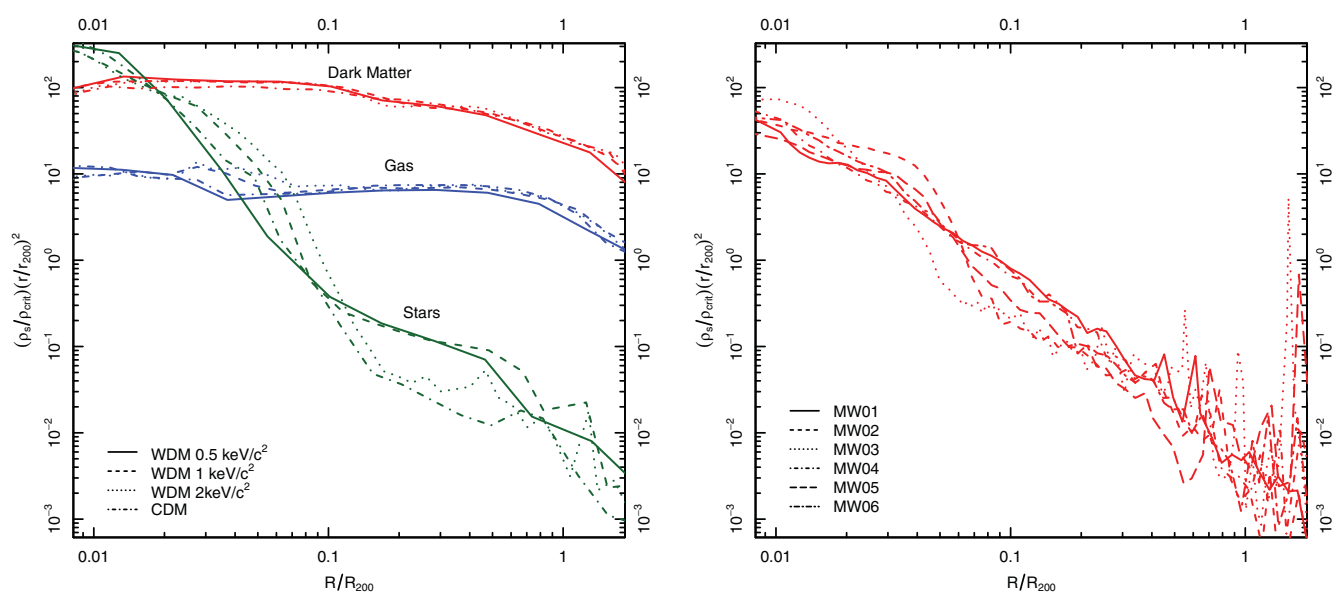

Figure 1. Spherically Averaged Mass Profiles at $z=\mathbf{0}$. Here we show spherically averaged mass density profiles of different components of the simulated galaxies. On the left we compare stars, dark matter, and gas inthe fiducial CDM run (dotted-dashed curves) and WDM $m_{\mathrm{WDM}}=2$, 1 , and $0.5 \mathrm{keV} / \mathrm{c}^{2}$ profiles (dotted, dashed, and solid curves). On the right we compare only stellar profiles for the 6 galaxies that form in the CDM model, all selected to form in low-density regions and all with $M_{200} \simeq 2 \times 10^{12} h^{-1} \mathrm{M}_{\odot}$ at $z=0$.

stellar mass or halo mass bins - as a testbed for dark matter, to carry out a statistical detection. Our results suggest that this will be challenging. However, the resolved ESC might also be used, by combining spatial, kinematic and metallicity substructure information to test dark matter; this will require the kind of statistical sample of Local Volume galaxies that will be accessible with LSST. Even if this remains a challenging test of dark matter, there is good reason to expect that we can use properties of the ESC to explore the mass assembly histories of galaxies, tracing merger and accretion events using material in the outer halo, and placing limits of the growth of galaxies in the context of their larger scale environment.

\section{References}

Bode, P., Ostriker, J. P., \& Turok, N. 2001, ApJ, 556, 93

Boylan-Kolchin, M., Bullock, J. S., \& Kaplinghat, M. 2011, MNRAS, 415, L40

Bullock, J. S. \& Johnston, K. V. 2005, ApJ, 635, 931

Cooper, A. P., Cole, S., Frenk, C. S., et al. 2010, MNRAS, 406, 744

Freeman, K. \& Bland-Hawthorn, J. 2002, ARA\&A, 40, 487

Helmi, A. 2008, A\&AR, 15, 145

Klypin A., Kravtsov A. V., Valenzuela O., \& Prada F. 1999, ApJ, 522, 82

Knebe, A., Arnold, B., Power, C., \& Gibson, B. K. 2008, MNRAS, 386, 1029

LSST Science Collaboration, Abell, P. A. Allison, J., et al. 2009, arXiv:0912.0201

Martínez-Delgado D., et al. 2010, AJ, 140, 962

Moore B., Ghigna S., Governato F., Lake G., Quinn T., Stadel J., \& Tozzi P. 1999, ApJ, 524, L19

Power, C. 2013, PASA, 30, 53

Power, C. \& Robotham, A. S. G. 2016, ApJ, 825, 31

Schneider, A., Anderhalden, D., Macciò, A. V., \& Diemand, J. 2014, MNRAS, 441, L6

Searle L., Zinn R. 1978, ApJ, 225, 357

Springel, V. 2005, MNRAS, 364, 1105

van Dokkum, P. G., Abraham, R., \& Merritt, A. 2014, ApJ(Letters), 782, L24 